PROCEEDINGS OF THE

AMERICAN MATHEMATICAL SOCIETY

Volume 127, Number 1, January 1999, Pages 205-210

S 0002-9939(99)05030-3

\title{
SOME REMARKS ON THE REAL RANK OF NON-UNITAL C*-ALGEBRAS
}

\author{
TAKASHI SAKAMOTO
}

(Communicated by David R. Larson)

\begin{abstract}
For a non-unital $\mathrm{C}^{*}$-algebra $A$, let $A^{\sim}$ be the $\mathrm{C}^{*}$-algebra obtained from $A$ by adjoining an identity. In this paper we show that $\operatorname{RR}\left(C_{0}(X) \otimes A\right)=\operatorname{RR}\left(C_{0}(X) \otimes A^{\sim}\right)$, where $X$ is a locally compact Hausdorff space with $\operatorname{RR}\left(C_{0}(X)\right) \leq 1$.
\end{abstract}

\section{InTRODUCTION}

For a unital $\mathrm{C}^{*}$-algebra $A$, L.G. Brown and G.K. Pedersen ([2]) define the real rank of $A$, denoted by $\operatorname{RR}(A)$, as the least integer $n$ such that for each $(n+1)$ tuple $\left(x_{0}, \cdots, x_{n}\right)$ of self-adjoint elements in $A$ and every $\varepsilon>0$, there exists an $(n+1)$-tuple $\left(y_{0}, \cdots, y_{n}\right)$ in $A_{s . a}$ such that $\sum_{j=0}^{n} y_{j}^{2}$ is invertible and

$$
\left\|x_{k}-y_{k}\right\|<\varepsilon \quad(k=0, \ldots, n) .
$$

M.A. Rieffel ([5]) defines the stable rank of $A$, denoted by $\operatorname{sr}(A)$, as the least integer $n$ such that for each $n$-tuple $\left(x_{1}, \cdots, x_{n}\right)$ of elements in $A$ and every $\varepsilon>0$, there exists an $n$-tuple $\left(y_{1}, \cdots, y_{n}\right)$ in $A$ such that $\sum_{j=1}^{n} y_{j}^{*} y_{j}$ is invertible and

$$
\left\|x_{k}-y_{k}\right\|<\varepsilon \quad(k=1, \ldots, n) .
$$

If $A$ is non-unital, then we define the real rank of $A$ and the stable rank of $A$ by $\operatorname{RR}\left(A^{\sim}\right)$ and $\operatorname{sr}\left(A^{\sim}\right)$ respectively, where the $\mathrm{C}^{*}$-algebra $A^{\sim}$ is obtained from $A$ by adjoining an identity.

In [2], L.G. Brown and G.K. Pedersen show that

$$
\operatorname{RR}(C(X))=\operatorname{dim} X
$$

for a unital commutative $\mathrm{C}^{*}$-algebra $C(X)$ of all the continuous functions on a compact Hausdorff space $X$, where $\operatorname{dim} X$ means the covering dimension of $X$. For every compact space $X$ and every paracompact $Y$, not both empty, we have

$$
\operatorname{dim}(X \times Y)=\operatorname{dim} X+\operatorname{dim} Y
$$

(see [3]). In [4], M. Nagisa, H. Osaka and N.C. Phillips show that

$$
\mathrm{RR}\left(C_{0}(X) \otimes A\right) \leq \operatorname{dim} X+\mathrm{RR}(A)
$$

Received by the editors May 9, 1997.

1991 Mathematics Subject Classification. Primary 46L05.

Key words and phrases. $\mathrm{C}^{*}$-algebra, real rank, stable rank.

(C)1999 American Mathematical Society 
for any $\mathrm{C}^{*}$-algebra and any locally compact, $\sigma$-compact, Hausdorff space, where $C_{0}(X)$ means all the continuous functions vanishing at infinity on $X$. We remark that a pathological phenomena occurs without the assumption of countability. Strictly speaking, for a non-unital $\mathrm{C}^{*}$-algebra $A$, they use the fact $\mathrm{RR}\left(C_{0}(X) \otimes A\right) \leq$ $\operatorname{RR}\left(C_{0}(X) \otimes A^{\sim}\right)$ and show that

$$
\mathrm{RR}\left(C_{0}(X) \otimes A^{\sim}\right) \leq \operatorname{dim} X+\mathrm{RR}(A) .
$$

In this note, we consider whether $\mathrm{RR}\left(C_{0}(X) \otimes A\right)$ differs from $\mathrm{RR}\left(C_{0}(X) \otimes A^{\sim}\right)$ or not. Our result is

$$
\operatorname{RR}\left(C_{0}(X) \otimes A\right)=\operatorname{RR}\left(C_{0}(X) \otimes A^{\sim}\right)
$$

for any non-unital $\mathrm{C}^{*}$-algebra $A$ and any locally compact Hausdorff space $X$ with $\operatorname{RR}\left(C_{0}(X)\right) \leq 1$.

\section{MAIN RESUlT}

We shall denote the one-point compactification of a locally compact Hausdorff space $X$ by $X \cup\{\infty\}$; then we can identify $C_{0}(X)^{\sim}$ and $C(X \cup\{\infty\})$. We shall also denote, by $C_{0}(X) \otimes A$, all the $A$-valued continuous functions vanishing at infinity on $X$. Then we can canonically identify $C_{0}(X, A)$ and the $\mathrm{C}^{*}$-tensor product $C_{0}(X) \otimes A$ of $C_{0}(X)$ and a $\mathrm{C}^{*}$-algebra $A$. We use $q_{A}$ to denote the natural quotient map $A^{\sim} \ni a+\lambda 1 \mapsto \lambda \in \mathbb{C} \cong A^{\sim} / A$.

The following lemma is shown in [2], [6].

Lemma 1. Let $0 \longrightarrow J \longrightarrow A \longrightarrow A / J \longrightarrow 0$ be an exact sequence of $C^{*}$ algebras. Then $\operatorname{RR}(A)=0$ if and only if $\operatorname{RR}(J)=\mathrm{RR}(A / J)=0$ and any projection in $A / J$ can be lifted to a projection in $A$.

The following proposition is essentially proved in [4]. Here, we extend the original one to the case that a $\mathrm{C}^{*}$-algebra $A$ is non-unital and a space $X$, instead of the interval, is general.

Proposition 2. Let $X$ be a locally compact Hausdorff space with $\mathrm{RR}\left(C_{0}(X)\right) \geq 1$. Then $\operatorname{RR}\left(C_{0}(X) \otimes A\right) \geq 1$ for any $C^{*}$-algebra $A$.

Proof. For any $x \in X$, the map $C_{0}(X) \otimes A\left(\simeq C_{0}(X, A)\right) \ni f \mapsto f(x) \in A$ is the surjective ${ }^{*}$-homomorphism. This implies that $\mathrm{RR}\left(C_{0}(X) \otimes A\right) \geq \mathrm{RR}(A)$. So we may assume that $\operatorname{RR}(A)=0$, and we consider the following exact sequence of $C^{*}$-algebras:

$$
0 \longrightarrow C_{0}(X) \otimes A \longrightarrow C(X \cup\{\infty\}) \otimes A \stackrel{\varphi}{\longrightarrow} A \longrightarrow 0,
$$

where $\varphi$ is defined by $\varphi(f)=f(\infty)$ for all $f \in C(X \cup\{\infty\}) \otimes A$. Clearly any projection in $A$ can be lifted to a projection in $C(X \cup\{\infty\}) \otimes A$. This means that $\operatorname{RR}(C(X \cup\{\infty\}) \otimes A)=0$, if $\operatorname{RR}\left(C_{0}(X) \otimes A\right)=0$. So it is enough to show that $\operatorname{RR}(C(X) \otimes A) \geq 1$ for a compact Hausdorff space $X$.

From the assumption $\operatorname{RR}(C(X)) \geq 1$, there exists a self-adjoint element $f$ in $C(X)$ and a positive number $\delta$ such that, if $g$ is in $C(X)_{\text {s.a. with }}\|f-g\|<\delta$, then $g(y)=0$ for some $y \in X$. Since $X$ is compact, we can find a positive constant $K$ such that $f(x)>-K$ for any $x \in X$. Let $a$ be a positive element in $A$ with $\|a\|=1$. We define a self-adjoint element $F \in(C(X) \otimes A)^{\sim}$ by

$$
F(x)=(f(x)+K) a-K 1 \quad(x \in X),
$$


where we identify $(C(X) \otimes A) \sim$ and the set $\left\{g \in C\left(X, A^{\sim}\right) \mid q_{A}(g(\cdot))\right.$ is a constant function on $X\}$. Let us denote, for any element $b$ in $A$, the set $\{\lambda \in \mathbb{C} \mid b-$ $\lambda 1$ is not invertible $\}$ by the symbol $S p(b)$. Then $f(x)=\max S p(F(x))$. In fact, by the positivity of $F(x)+K 1$, we have

$$
\begin{aligned}
\max S p(F(x))+K & =\max S p(F(x)+K 1)=\|F(x)+K 1\| \\
& =\|(f(x)+K) a\|=f(x)+K .
\end{aligned}
$$

If $G$ is a self-adjoint element in $(C(X) \otimes A)^{\sim}$ with $\|F-G\|<\delta$, then we have $\max S p(G(\cdot)) \in C(X)_{s . a .}$ and $\|f-\max S p(G(\cdot))\|<\delta$. Therefore $\max S p(G(y))=0$ for some $y \in X$, that is, $G$ is not invertible. This implies $\operatorname{RR}(C(X) \otimes A) \geq 1$.

Theorem 3. Let $X$ be a locally compact Hausdorff space with $\operatorname{RR}\left(C_{0}(X)\right) \leq 1$. Then $\operatorname{RR}\left(C_{0}(X) \otimes A\right)=\operatorname{RR}\left(C_{0}(X) \otimes A^{\sim}\right)$ for any non-unital $C^{*}$-algebra $A$.

Proof. We shall prove only the case that $X$ is non-compact. We can similarly prove the case that $X$ is compact.

For a locally compact, non-compact Hausdorff space $X$, we can identify $\left(C_{0}(X)\right.$ $\left.\otimes A^{\sim}\right)^{\sim}$ and

$$
\left\{F \in C\left(X \cup\{\infty\}, A^{\sim}\right) \mid F(\infty)=q_{A}(F(\infty)) 1\right\} .
$$

In this identification, we can see $\left(C_{0}(X) \otimes A\right)^{\sim}$ as

$$
\left\{F \in C\left(X \cup\{\infty\}, A^{\sim}\right) \mid F(\infty)=q_{A}(F(x)) 1, \quad \text { for all } x \in X\right\} .
$$

We consider the following split exact sequence of $\mathrm{C}^{*}$-algebras:

$$
0 \longrightarrow C_{0}(X) \otimes A \longrightarrow\left(C_{0}(X) \otimes A^{\sim}\right)^{\sim} \underset{j}{\stackrel{\pi}{\longrightarrow}} C(X \cup\{\infty\}) \longrightarrow 0,
$$

where $\pi(F)(x)=q_{A}(F(x))$ and $j(f)(x)=f(x) 1$ for $F \in\left(C_{0}(X) \otimes A^{\sim}\right)^{\sim}, f \in$ $C_{0}(X \cup\{\infty\})$ and $x \in X \cup\{\infty\}$. Clearly we have

$$
\operatorname{RR}\left(C_{0}(X) \otimes A\right) \leq \operatorname{RR}\left(C_{0}(X) \otimes A^{\sim}\right) .
$$

At first, we examine the case that the real rank of $C_{0}(X) \otimes A$ is zero. By Proposition 2, we have $\operatorname{RR}\left(C_{0}(X)\right)=0$ and it is clear that any projection in $C(X \cup\{\infty\})$ can be lifted to a projection in $\left(C_{0}(X) \otimes A^{\sim}\right)^{\sim}$. So we get that $\operatorname{RR}\left(\left(C_{0}(X) \otimes A^{\sim}\right)\right)=0$ by Lemma 1 .

In the case that $\operatorname{RR}\left(C_{0}(X) \otimes A\right)=n \geq 1$, we shall show that $\operatorname{RR}\left(C_{0}(X) \otimes A^{\sim}\right) \leq$ $n$. Let $F_{0}, F_{1}, \ldots, F_{n}$ be self-adjoint elements in $\left(C_{0}(X) \otimes A^{\sim}\right)^{\sim}$, and set

$$
g_{0}=\pi\left(F_{0}\right), g_{1}=\pi\left(F_{1}\right), f_{2}=\pi\left(F_{2}\right), \ldots, f_{n}=\pi\left(F_{n}\right) .
$$

Since $\operatorname{RR}(C(X \cup\{\infty\})) \leq 1$, for any $\varepsilon>0$ we can choose $f_{0}, f_{1} \in C(X \cup\{\infty\})_{\text {s.a. }}$. such that $\left\|g_{0}-f_{0}\right\|<\varepsilon,\left\|g_{1}-f_{1}\right\|<\varepsilon$ and $f_{0}^{2}+f_{1}^{2}>0$. We consider an element $\Delta$ in $\mathbb{M}_{n+1}(C(X \cup\{\infty\}))$ as follows:

$$
\Delta=\left(\begin{array}{ccccccc}
\frac{f_{0}}{k_{0}} & \frac{f_{1}}{k_{0}} & \frac{f_{2}}{k_{0}} & \frac{f_{3}}{k_{0}} & \ldots & \frac{f_{n-1}}{k_{0}} & \frac{f_{n}}{k_{0}} \\
-\frac{f_{1}}{k_{1}} & \frac{f_{0}}{k_{1}} & 0 & 0 & \ldots & 0 & 0 \\
-\frac{f_{0} f_{2}}{k_{2}} & -\frac{f_{1} f_{2}}{k_{2}} & \frac{f_{0}^{2}+f_{1}^{2}}{k_{2}} & 0 & \ldots & 0 & 0 \\
\vdots & \vdots & \vdots & \vdots & \ddots & \vdots & \vdots \\
-\frac{f_{0} f_{n}}{k_{n}} & -\frac{f_{1} f_{n}}{k_{n}} & -\frac{f_{2} f_{n}}{k_{n}} & -\frac{f_{3} f_{n}}{k_{n}} & \ldots & -\frac{f_{n-1} f_{n}}{k_{n}} & \frac{\sum_{k=0}^{n-1} f_{k}^{2}}{k_{n}}
\end{array}\right),
$$


where $k_{0}=\sqrt{f_{0}^{2}+f_{1}^{2}+\cdots+f_{n}^{2}}, k_{1}=\sqrt{f_{0}^{2}+f_{1}^{2}}$ and

$$
k_{l}=\sqrt{\left(f_{0}^{2}+\cdots+f_{l-1}^{2}\right)\left(f_{0}^{2}+\cdots+f_{l}^{2}\right)} \quad(2 \leq l \leq n) .
$$

Then we have

$$
{ }^{t} \Delta \Delta=I_{n+1} \quad \text { and } \quad \frac{1}{k_{0}} \Delta\left(\begin{array}{c}
f_{0} \\
f_{1} \\
\vdots \\
f_{n}
\end{array}\right)=\left(\begin{array}{c}
1 \\
0 \\
\vdots \\
0
\end{array}\right) .
$$

We define self-adjoint elements $G_{0}, G_{1}, \ldots, G_{n}$ in $\left(C_{0}(X) \otimes A^{\sim}\right)^{\sim}$ by the following relation:

$$
\left(j \otimes i d_{n+1}\right)\left(\frac{1}{k_{0}} \Delta\right)\left(\begin{array}{c}
F_{0}-j\left(g_{0}\right)+j\left(f_{0}\right) \\
F_{1}-j\left(g_{1}\right)+j\left(f_{1}\right) \\
F_{2} \\
\vdots \\
F_{n}
\end{array}\right)=\left(\begin{array}{c}
G_{0} \\
G_{1} \\
G_{2} \\
\vdots \\
G_{n}
\end{array}\right) .
$$

Then we can easily check

$$
q_{A}\left(G_{0}(x)\right)=1, q_{A}\left(G_{1}(x)\right)=0, \ldots, q_{A}\left(G_{n}(x)\right)=0
$$

for any $x \in X \cup\{\infty\}$, that is, $G_{0}, \ldots, G_{n}$ belong to $\left(C_{0}(X) \otimes A\right)^{\sim}$. So we can choose self-adjoint elements $\tilde{G}_{0}, \tilde{G}_{1}, \ldots, \tilde{G}_{n}$ in $\left(C_{0}(X) \otimes A\right)^{\sim}$ satisfying $\left\|G_{l}-\tilde{G}_{l}\right\|<$ $\left(1 /\left\|k_{0}\right\|\right) \varepsilon$ for $0 \leq l \leq n$, and $\sum_{l=0}^{n}\left(\tilde{G}_{l}\right)^{2}$ is invertible. If we put

$$
\left(\begin{array}{c}
\tilde{F}_{0} \\
\tilde{F}_{1} \\
\vdots \\
\tilde{F}_{n}
\end{array}\right)=\left(j \otimes i d_{n+1}\right)\left(k_{0}{ }^{t} \Delta\right)\left(\begin{array}{c}
\tilde{G}_{0} \\
\tilde{G}_{1} \\
\vdots \\
\tilde{G}_{n}
\end{array}\right)
$$

then we get $\tilde{F}_{l} \in\left(C_{0}(X) \otimes A^{\sim}\right)^{\sim}$ with $\left\|F_{l}-\tilde{F}_{l}\right\|<\varepsilon$ for $0 \leq l \leq n$, and $\sum_{l=0}^{n}\left(\tilde{F}_{l}\right)^{2}=$ $j\left(k_{0}^{2}\right) \cdot \sum_{l=0}^{n}\left(\tilde{G}_{k}\right)^{2}$ is invertible.

In the above theorem, for a space $X$ with $\mathrm{RR}\left(C_{0}(X)\right) \geq 2$, it is not true that $\operatorname{RR}\left(C_{0}(X) \otimes A\right)=\operatorname{RR}\left(C_{0}(X) \otimes A^{\sim}\right)$ in general. For example, let us denote by $I$ the $[0,1]$-interval and by $\mathbb{K}$ the $\mathrm{C}^{*}$-algebra of all the compact operators. Then we have

$$
\mathrm{RR}(C(I \times I) \otimes \mathbb{K})=1
$$

(see [1], [2]). But, by the fact $C(I \times I) \otimes \mathbb{K}^{\sim} / C(I \times I) \otimes \mathbb{K} \cong C(I \times I)$, we have

$$
\operatorname{RR}\left(C(I \times I) \otimes \mathbb{K}^{\sim}\right) \geq 2 .
$$

We can apply the above argument for real rank to that for stable rank. Then we have the following statement:

Proposition 4. Let $X$ be a locally compact Hausdorff space, and $A$ a non-unital $C^{*}$-algebra.

(1) If $\operatorname{sr}\left(C_{0}(X)\right)=1$, then

$$
\operatorname{sr}\left(C_{0}(X) \otimes A\right)=\operatorname{sr}\left(C_{0}(X) \otimes A^{\sim}\right) .
$$


(2) If $\operatorname{sr}\left(C_{0}(X)\right)=2$ and $\operatorname{sr}\left(C_{0}(X) \otimes A\right) \geq 2$, then

$$
\operatorname{sr}\left(C_{0}(X) \otimes A\right)=\operatorname{sr}\left(C_{0}(X) \otimes A^{\sim}\right) .
$$

Proof. We use the identification as stated in the proof of Theorem 3. From the split exact sequence of $\mathrm{C}^{*}$-algebras

$$
0 \longrightarrow C_{0}(X) \otimes A \longrightarrow\left(C_{0}(X) \otimes A^{\sim}\right)^{\sim} \underset{j}{\stackrel{\pi}{\longrightarrow}} C(X \cup\{\infty\}) \longrightarrow 0,
$$

we have $\operatorname{sr}\left(C_{0}(X) \otimes A\right) \leq \operatorname{sr}\left(C_{0}(X) \otimes A^{\sim}\right)$.

First, we examine the case that $\operatorname{sr}\left(C_{0}(X)\right) \leq 2$ and $\operatorname{sr}\left(C_{0}(X) \otimes A\right) \geq 2$. Suppose that $\operatorname{sr}\left(C_{0}(X) \otimes A\right)=n$. We shall show that $\operatorname{sr}\left(C_{0}(X) \otimes A^{\sim}\right) \leq n$. Let $F_{1}, F_{2}, \ldots, F_{n}$ be elements in $\left(C_{0}(X) \otimes A^{\sim}\right)^{\sim}$, and set

$$
g_{1}=\pi\left(F_{1}\right), g_{2}=\pi\left(F_{2}\right), f_{3}=\pi\left(F_{3}\right), \ldots, f_{n}=\pi\left(F_{n}\right) .
$$

Since $\operatorname{sr}(C(X \cup\{\infty\})) \leq 2$, for any $\varepsilon>0$ we can choose $f_{1}, f_{2} \in C(X \cup\{\infty\})$ such that $\left\|g_{1}-f_{1}\right\|<\varepsilon,\left\|g_{2}-f_{2}\right\|<\varepsilon$ and $\left|f_{1}\right|^{2}+\left|f_{2}\right|^{2}>0$. We consider an element $\Delta$ in $\mathbb{M}_{n}(C(X \cup\{\infty\}))$ as follows:

$$
\Delta=\left(\begin{array}{ccccccc}
\frac{\overline{f_{1}}}{k_{1}} & \overline{f_{2}} & \overline{f_{3}} & \overline{f_{4}} & \ldots & \frac{\overline{f_{n-1}}}{k_{1}} & \frac{\overline{f_{n}}}{k_{1}} \\
-\frac{\overline{k_{2}}}{k_{2}} & \overline{f_{1}} & 0 & 0 & \ldots & 0 & 0 \\
-\frac{\overline{f_{1} f_{3}}}{k_{3}} & -\frac{f_{2} f_{3}}{k_{3}} & \frac{\left|f_{1}\right|^{2}+\left|f_{2}\right|^{2}}{k_{3}} & 0 & \ldots & 0 & 0 \\
\vdots & \vdots & \vdots & \vdots & \ddots & \vdots & \vdots \\
-\frac{\overline{f_{1}} f_{n}}{k_{n}} & -\frac{\overline{f_{2}} f_{n}}{k_{n}} & -\frac{\overline{f_{3}} f_{n}}{k_{n}} & -\frac{\overline{f_{4}} f_{n}}{k_{n}} & \ldots & -\frac{\overline{f_{n-1}} f_{n}}{k_{n}} & \frac{\sum_{k=1}^{n-1}\left|f_{k}\right|^{2}}{k_{n}}
\end{array}\right),
$$

where $\bar{f}$ means the complex conjugate of $f$,

$$
k_{1}=\sqrt{\left|f_{1}\right|^{2}+\cdots+\left|f_{n}\right|^{2}}, \quad k_{2}=\sqrt{\left|f_{1}\right|^{2}+\left|f_{2}\right|^{2}}
$$

and

$$
k_{l}=\sqrt{\left(\left|f_{1}\right|^{2}+\cdots+\left|f_{l-1}\right|^{2}\right)\left(\left|f_{1}\right|^{2}+\cdots+\left|f_{l}\right|^{2}\right)} \quad(3 \leq l \leq n) .
$$

Then we have

$$
{ }^{t} \bar{\Delta} \Delta=I_{n} \quad \text { and } \quad \frac{1}{k_{1}} \Delta\left(\begin{array}{c}
f_{1} \\
f_{2} \\
\vdots \\
f_{n}
\end{array}\right)=\left(\begin{array}{c}
1 \\
0 \\
\vdots \\
0
\end{array}\right) .
$$

For elements $G_{1}, G_{2}, \ldots, G_{n}$ in $\left(C_{0}(X) \otimes A\right)^{\sim}$ which are defined by

$$
\left(j \otimes i d_{n}\right)\left(\frac{1}{k_{1}} \Delta\right)\left(\begin{array}{c}
F_{1}-j\left(g_{1}\right)+j\left(f_{1}\right) \\
F_{2} \\
F_{3} \\
\vdots \\
F_{n}
\end{array}\right)=\left(\begin{array}{c}
G_{1} \\
G_{2} \\
G_{3} \\
\vdots \\
G_{n}
\end{array}\right),
$$

we can choose elements $\tilde{G}_{1}, \tilde{G}_{2}, \ldots, \tilde{G}_{n}$ in $\left(C_{0}(X) \otimes A\right)^{\sim}$ satisfying $\left\|G_{l}-\tilde{G}_{l}\right\|<$ $\left(1 /\left\|k_{1}\right\|\right) \varepsilon$ for $1 \leq l \leq n$, and $\sum_{l=1}^{n}\left(\tilde{G}_{l}\right)^{2}$ is invertible. Then we can get the desired 
approximants $\tilde{F}_{1}, \ldots, \tilde{F}_{n}$ by the relation

$$
\left(\begin{array}{c}
\tilde{F}_{1} \\
\tilde{F}_{2} \\
\vdots \\
\tilde{F}_{n}
\end{array}\right)=\left(j \otimes i d_{n}\right)\left(k_{1}{ }^{t} \bar{\Delta}\right)\left(\begin{array}{c}
\tilde{G}_{1} \\
\tilde{G}_{2} \\
\vdots \\
\tilde{G}_{n}
\end{array}\right) .
$$

Next, we consider the case that $\operatorname{sr}\left(C_{0}(X) \otimes A\right)=1$ and $\operatorname{sr}\left(C_{0}(X)\right)=1$. The above argument can be applied to this case. There, $\Delta$ is a $1 \times 1$ matrix $\frac{f}{|f|}$ for an invertible element $f$ in $C(X \cup\{\infty\})$.

We also remark that

$$
\operatorname{sr}\left(C\left(I^{4}\right) \otimes \mathbb{K}\right)=2 \text { and } \operatorname{sr}\left(C\left(I^{4}\right) \otimes \mathbb{K}^{\sim}\right) \geq \operatorname{sr}\left(C\left(I^{4}\right)\right)=3 .
$$

So, in the case $\operatorname{sr}\left(C_{0}(X)\right) \geq 3$,

$$
\operatorname{sr}\left(C_{0}(X) \otimes A\right)=\operatorname{sr}\left(C_{0}(X) \otimes A^{\sim}\right)
$$

does not hold in general.

In [4], the following fact is proved: if $\operatorname{sr}\left(C\left(I^{n}\right) \otimes A\right)=1$ for a unital $\mathrm{C}^{*}$-algebra $A$, then we have $n=0$ or 1 (i.e. $\operatorname{sr}\left(C\left(I^{n}\right)\right)=1$ ). For a non-unital $\mathrm{C}^{*}$-algebra $A$ and a locally compact Hausdorff space $X$, we do not know whether $\operatorname{sr}\left(C_{0}(X) \otimes A\right)=1$ implies $\operatorname{sr}\left(C_{0}(X)\right)=1$ or not.

\section{ACKNowledgement}

I would like to express my deep gratitude to M. Nagisa for his help with this paper.

\section{REFERENCES}

1. E. J. Beggs and D. E. Evans, The real rank of matrix valued functions, Internat. J. Math. 2 (1991), 131-138. MR 92e:46114

2. L. G. Brown and G. K. Pedersen, $C^{*}$-algebras of real rank zero, J. Funct. Anal. 99 (1991), 131-149. MR 92m:46086

3. R. Engelking, Theory of Dimensions Finite and Infinite, Sigma Ser. in Pure Math. vol.10, Heldermann Verlag, 1995. MR 97j:54033

4. M. Nagisa, H. Osaka and N. C. Phillips, Rank of non-commutative algebras of continuous $C^{*}$-algebra valued functions over the interval, Preliminary version.

5. M. A. Rieffel, Dimension and stable rank in the K-theory of $C^{*}$-algebras, Proc. London Math. Soc. 46 (1987), 301-333. MR 84g:46085

6. S. Zhang, Certain $C^{*}$-algebras with real rank zero and their corona and multiplier algebras. Part I, Pacific J. Math. 155 (1992), 169-197. MR 94i:46093

Department of Mathematics and Informatics, Graduate School of Science and Technology, Chiba University, 1-33, Yayoi-Cho, Inage-Ku, Chiba 263-8522, Japan

E-mail address: msakamot@math.s.chiba-u.ac.jp 